\title{
Psoriasis Exacerbates the State of Insulin Resistance in Patients with Type 2 Diabetes
}

\author{
Song Wen ${ }^{1, *}$ \\ Congying Liu ${ }^{1, *}$ \\ Yanyan Li' \\ Junhong Pan' \\ Thiquynhnga Nguyen' \\ Ligang Zhou ${ }^{1,2}$
}

'Department of Endocrinology, Shanghai Pudong Hospital, Fudan University, Shanghai, 201399, People's Republic of China; ${ }^{2}$ Shanghai Key Laboratory of Vascular Lesions Regulation and

Remodeling, Shanghai Pudong Hospital, Fudan University Pudong Medical Center, Shanghai, 201399, People's Republic of China

*These authors contributed equally to this work
Correspondence: Ligang Zhou Department of Endocrinology, Shanghai Pudong Hospital, Fudan University, Shanghai, 201399, People's Republic of China

Tel +86I36II9276I6

Email zhouligang@yahoo.com
Purpose: Although psoriasis ( $\mathrm{PsO}$ ) is highly associated with insulin resistance (IR), the role of $\mathrm{PsO}$ on activity of insulin secretion or its action in diabetic patients has not been explored. Materials and Methods: In-patient data on type 2 diabetes (T2D) with or without PsO from 2016-2019 in our hospital were analyzed. Data for 42 diabetic patients with PsO were compared with that of the control group (T2D only). Blood examinations with reference to the levels of fasting blood glucose, C-peptide, insulin, HbAlc, plasma lipids, lipoproteins, and kidney function were explored. HOMA-IR and HOMA- $\beta$ models were established to explore IR and pancreatic $\beta$-cell function.

Results: HOMA-IR level was significantly higher $(P=0.0003<0.05)$ in patients with PsO compared with the controls. Although the durations of diabetes in patients with PsO were significantly shorter compared with that of patients with diabetes only $(P=0.012<0.05)$, analysis of mean BMI, eGFR, plasma lipids, and lipoprotein showed no significant differences. Analysis of the level of fasting glucose and HOMA- $\beta$ showed no statistical differences between the two groups. On the other hand, the levels of C-peptide of PsO group were significantly high in both fasting state $(P=0.0182<0.05)$ and after glucose challenge $(P=0.0011<0.01)$.

Conclusion: The findings of this study show that under the same fasting conditions, patients with $\mathrm{PsO}$ may have relatively preserved pancreatic $\beta$-cell function, and PsO significantly increases IR.

Keywords: psoriasis, type 2 diabetes mellitus, IR, $\beta$-cell function

\section{Introduction}

Psoriasis ( $\mathrm{PsO})$ is a common chronic inflammatory skin disease. ${ }^{1}$ Individuals with $\mathrm{PsO}$ are at higher risk of developing other chronic and severe health diseases including psoriatic arthritis, metabolic syndrome, cardiovascular disorders, anxiety and depression, non-alcoholic fatty liver disease, Crohn's disease, and lymphoma. ${ }^{2,3}$ Diabetes is a group of metabolic diseases characterized by chronic hyperglycemia due to absolute or relative deficiency of insulin secretion and IR. ${ }^{4}$ Currently, there is increased interest in exploring the relationship between the PsO and T2D. Previous epidemiological studies report that the risk of diabetes in patients with $\mathrm{PsO}$ is significantly higher compared with that in individuals without PsO. Studies project additional 125,650 new diagnoses in T2D per year worldwide in patients with psoriasis compared with those without PsO. ${ }^{5}$ In addition, severity of $\mathrm{PsO}$ is correlated with the risk of T2D. ${ }^{6}$ Notably, PsO and T2D show genetic and pathological similarities and common targets, such as CDKAL1, PSORS2, PSORS3, PSORS4, which are susceptible genes for PsO and are also associated 
with $\mathrm{T} 2 \mathrm{D} .^{7,8}$ Studies report that inflammatory cytokines (such as TNF- $\alpha$ and IL-17), ${ }^{9}$ adipocytokines (such as resistin and chemerin), ${ }^{10}$ and gastrointestinal peptide (Incretins) $)^{11}$ are associated with pathology of both PsO and T2D. Furthermore, anti-inflammatory or immunerestricting therapy are effective in modulating glucose metabolism in T2D and inflammation in $\mathrm{PsO}^{12,13}$ However, the effect of PsO on insulin activity in T2D has not been explored. Therefore, this study explored changes in IR by determining HOMA-IR level in diabetic patients with $\mathrm{PsO}$, and provides a possible strategy for effective management of glucose homeostasis in diabetic patients with $\mathrm{PsO}$.

\section{Methods}

\section{Source of In-Patient Data}

Patients' data were obtained from an in-patient information system of Shanghai Pudong Hospital. Forty-two patients with a T2D history hospitalized in the Department of Endocrinology diagnosed with PsO were included in this study. Exclusion criteria included patients who are prediabetes, have type 1 diabetes, have latent autoimmune diabetes for adults (LADA), other special types of diabetes, gestational diabetes, presence of chronic inflammatory diseases other than $\mathrm{PsO}$, severe anemia, pregnancy, or breastfeeding. The control group comprised patients with a history of T2D and without PsO, and met the exclusion criteria used for the PsO group. Diagnoses for T2D were based on the guidelines reported by the World Health Organization (WHO) in 1999.

\section{Baseline Information Evaluation}

Baseline data obtained included gender, age, duration of T2D, history of drug use, BMI, and related contents (Table 1). Treatments approaches for $\mathrm{PsO}$ included ultraviolet radiation, urea ointment, budesonide ointment, methotrexate, and vitamin A.

\section{Blood Glucose Level, and Evaluation of Lipid Metabolism and Kidney Function}

Fasting plasma glucose (FPG), 120 minute post-meal plasma glucose (PPG), HbA1c, fasting C-peptide (FCP), 120 minute post-meal C-peptide (PCP), fasting insulin (FIN), and 120 minute post-meal insulin (PIN) were determined. Lipids test included determination of total triglyceride (TG), total cholesterol (TC), High density lipoprotein (HDL), and Low density lipoprotein (LDL).
Table I Baseline Information of Diabetic Patients with and without $\mathrm{PsO}$

\begin{tabular}{|l|c|c|c|}
\hline & PsO+T2D & Controls & $P$ \\
\hline Patients (n) & 42 & 99 & $/$ \\
\hline Gender (Male/Female) & $31 / 1 I^{* *}$ & $47 / 52$ & 0.003 \\
\hline Age (years) & $62.00 \pm 16.86$ & $\begin{array}{c}64.28 \\
\pm 13.06\end{array}$ & 0.436 \\
\hline Duration (years) & $7.73 \pm 7.32 *$ & $\begin{array}{c}11.38 \\
\pm 7.94\end{array}$ & 0.012 \\
\hline T2D drugs (Insulin/OADs $\S /$ & $11 / 15 / 6 * * *$ & $55 / 37 / 0$ & $<0.001$ \\
combination) & $1 I^{\#}$ & $/$ & $/$ \\
\hline PsO drugs (n) & $26.10 \pm 4.44$ & $\begin{array}{c}25.39 \\
\pm 3.65\end{array}$ & 0.415 \\
\hline BMI (kg/m ${ }^{2}$ ) & & $\begin{array}{c}9.052 \\
\pm 2.099\end{array}$ & 0.360 \\
\hline HbAlc (\%) & $8.678 \pm 2.383$ & \\
\hline
\end{tabular}

Notes: $* P<0.05 ; * * P<0.01$; $* * * P<0.001$; ${ }^{\S}$ Oral antidiabetic drugs for $\mathrm{PsO}$ : eight patients used metformin, five used $\alpha$-glucosidase inhibitors, nine used sulfonylureas, none used Glinide, two used Thiazolidinedione (TZDs), and none used dipeptidyl peptidase IV (DPP-IV) inhibitors, one patient was unknown; combinations of insulin and OADs: three patients used metformin and insulin, one used sulfonylureas and insulin, one used Glinide and insulin, one used TZDs and insulin, one patient was unknown. For controls using OADs: 29 patients used metformin, 12 used $\alpha$ glucosidase inhibitors, 22 used sulfonylureas, one used Glinide, nine used Thiazolidinedione (TZDs), and none used dipeptidyl peptidase IV (DPP-IV) inhibitors, one patient was unknown. "The II patients with $\mathrm{PsO}+\mathrm{T} 2 \mathrm{D}$ received $\mathrm{PsO}$ drugs, including two patients with only oral retinoic acid therapy, one patient administered with only oral methotrexate, one patient using only urea ointment, one using budesonide cream combined with oral retinoic acid and ultraviolet therapy, and six using traditional Chinese medication therapy.

Abbreviations: PsO, psoriasis; T2D, type 2 diabetes; OADs, oral antidiabetic medications; BMI, body mass index; HbAlc, hemoglobin Alc.

In addition, kidney function tests including eGFR were performed.

\section{Establishment of HOMA-IR and HOMA- $\beta$ Models}

Fasting glucose and fasting C-peptide were used to perform homeostasis model assessment to quantify and compare IR and $\beta$-cell function between the $\mathrm{PsO}$ group and control group. HOMA-IR and HOMA- $\beta$ models were developed using a calculator obtained from the University of Oxford database (http://www.dtu.ox.ac.uk/).

\section{Statistical Analyses}

Statistics analyses were performed in SPSS (IBM, version 24.0) and Prism (GraphPad, version 8.0). Independent $t$-tests were used to compare differences between BMI, duration of diabetes and eGFR of HOMA-IR and HOMA- $\beta$ groups. 
Two-way ANOVA was used to compare the levels of plasma lipids and lipoprotein, glucose, insulin, C-peptide change between the two groups. Statistical significance was determined at $P \leq 0.05$ level for all analyses.

\section{Results}

\section{IR Was Significantly Higher in the PsO Group Compared with the T2D Group without PsO}

Analysis showed significantly higher levels of HOMA-IR, FPG, and C-peptide in the PsO group (PsO) compared with the levels in the control group (T2D) $(M e a n \pm S D$ : PsO vs T2D: $5.863 \pm 21.070$ vs $1.042 \pm 0.763, P=0.0003$, PsO: n=42; T2D: n=99; Figure 1).

\section{BMI, Estimated Renal Glomerular}

Filtration Rate (eGFR), Plasma Lipids and Lipoproteins in the PsO Were Not Statistically Different from Those in the Control Group

Analyses of anthropometry of BMI, and biochemistry tests of renal eGFR, plasma lipids and lipoprotein, showed no statistical differences between the two groups (Mean $\pm \mathrm{SD}$ : PsO+T2D vs controls: BMI: $26.10 \pm 4.44$ vs $25.39 \pm 3.65 \mathrm{~kg} /$ $\mathrm{m}^{2}, P=0.415$; eGFR: $81.93 \pm 33.84$ vs $89.06 \pm 25.59 \mathrm{~mL} /$ $\min * 1.73 \mathrm{~m}^{2}, P=0.2673$; TG: $2.19 \pm 1.15$ vs $1.79 \pm 1.52$ $\mathrm{mmol} / \mathrm{L}, P=0.1552$; TC: $4.09 \pm 0.98$ vs $4.20 \pm 1.17 \mathrm{mmol} / \mathrm{L}$,

\section{HOMA-IR}

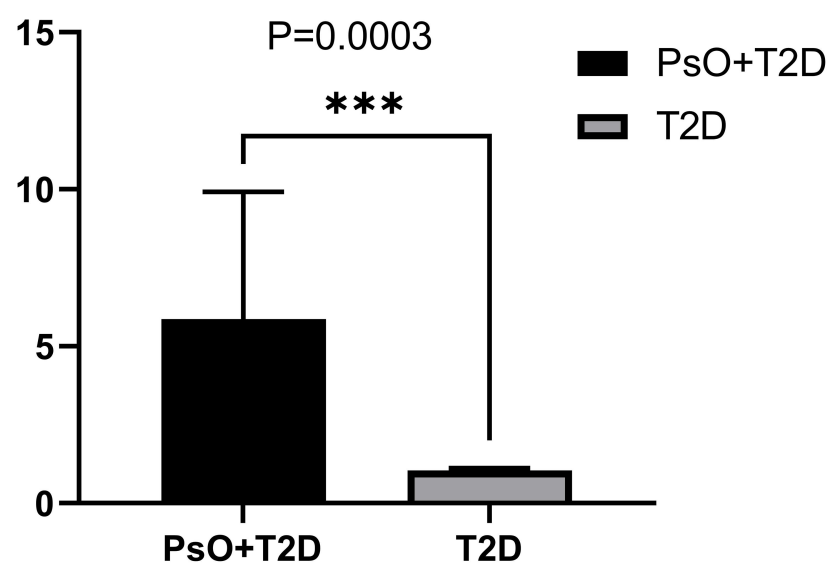

Figure I Comparison of IR represented by HOMA-IR in the $\mathrm{PsO}$ group and controls.

Note: $* * * P=0.0003$.

Abbreviations: HOMA-IR, homeostasis model assessment of IR; T2D, type 2 diabetes mellitus control group; $\mathrm{PsO}+\mathrm{T} 2 \mathrm{D}$, group of type 2 diabetes mellitus with psoriasis.
$P=0.9696 ; \quad$ LDL: $\quad 2.38 \pm 0.91 \quad$ vs $\quad 2.54 \pm 0.94 \quad \mathrm{mmol} / \mathrm{L}$, $P=0.8738$; HDL: $0.93 \pm 0.33 \mathrm{mmol} / \mathrm{L}$ vs $1.04 \pm 0.29 \mathrm{mmol} /$ $\mathrm{L}, P=0.9654$ ) (Figure 2).

\section{Analyses of Pancreatic $\beta$-Cell Function Showed Significant Differences in Levels of C-Peptide, but Not in HOMA- $\beta$, Plasma Glucose, and Insulin between the $\mathrm{PsO}$ and Control Group}

C-peptide level in both fasting state and after 120 minutes was significantly higher in the $\mathrm{PsO}$ group compared with the level for the control group ( 0 minutes: $P=0.0182<0.05$; 120 minutes: $P=0.0011<0.01$; PsO: $\mathrm{n}=42$; controls: $\mathrm{n}=99$ ), whereas the HOMA- $\beta$, plasma glucose level, and insulin level showed no significant disparity in the PsO group compared with the control (Mean \pm SD:HOMA- $\beta$ : PsO vs controls: $\quad 47.844 \pm 33.839 \quad$ vs $\quad 41.296 \pm 30.774$; $P=0.3387>0.05$; $\quad$ glucose level: 0 minutes: $P=0.4805>0.05 ; 120$ minutes: $P=0.2187>0.05$; insulin level: 0 minutes: $P=0.9984>0.05 ; 120$ minutes: $P=0.3479>0.05$, PsO: $\mathrm{n}=42$; controls: $\mathrm{n}=99$; Table 2 and Figure 3).

\section{Discussion}

PsO and type 2 diabetes mellitus (T2D) are chronic diseases that share common mechanisms including chronic inflammation in multiple organs and tissue. Studies report that $\mathrm{PsO}$ is highly correlated with metabolic syndrome, obesity, IR, diabetes, atherosclerosis, and cardiovascular diseases. ${ }^{1}$ However, the effect of $\mathrm{PsO}$ on glucose metabolism in T2D has not been explored. Therefore, the current study sought to retrospectively explore the effect of $\mathrm{PsO}$ on metabolic profiles including plasma glucose, C-peptide, insulin, and lipids levels. In addition, HOMA models were established to explore IR and $\beta$-cell function in the PsO and the control groups. Gender proportion, duration of diabetes, proportion of drug administration in T2D, HOMA-IR, and C-peptide level showed significant differences between the two groups. The findings of this study show the role of $\mathrm{PsO}$ in pathophysiology of T2D, and provide a possible strategy for treatment of diabetic patients with PsO.

Analysis showed that the prevalence of $\mathrm{PsO}$ was higher in male diabetic patients compared with female diabetic patients (Table 1), which is consistent with findings from a previous epidemiological study. ${ }^{14}$ Previous studies report that systemic therapy results in better outcome of PsO 
A
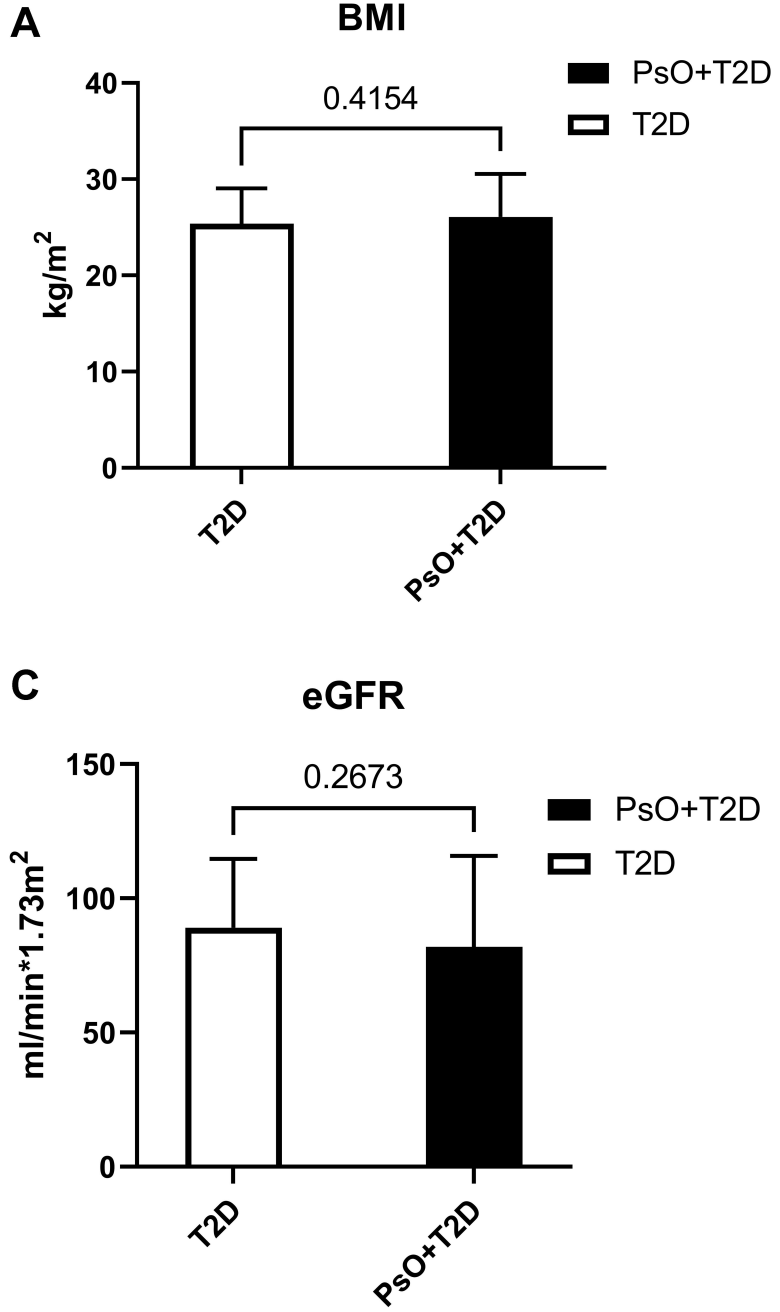

B Duration

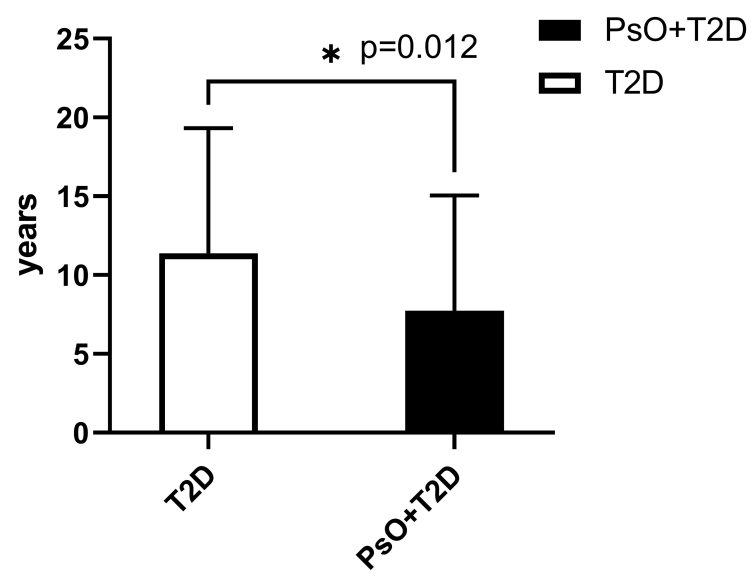

Plasma lipids and lipoprotein

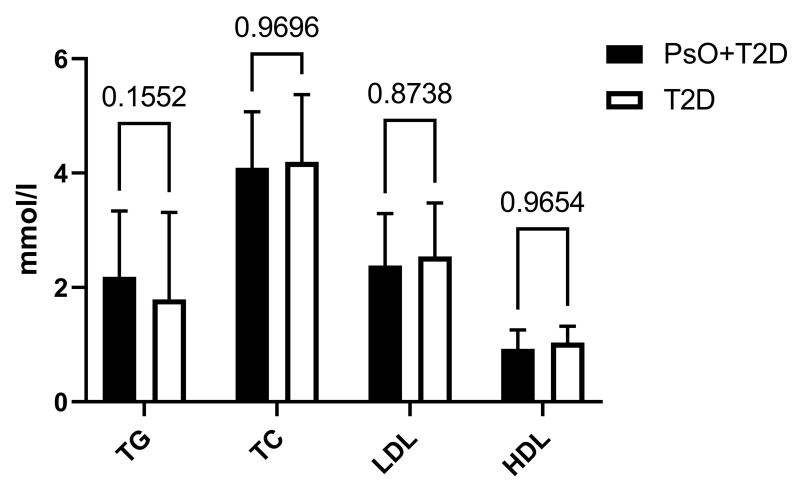

Figure 2 BMI (A), duration of diabetes (B), eGFR (C), plasma lipids and lipoproteins (D) in the PsO group and diabetic patients without psoriasis. Note: $* P<0.05$.

Abbreviations: BMI, body mass index; eGFR, estimated glomerular filtration rate; TG, total triglyceride; TC, total cholesterol; LDL, low density lipoprotein; HDL, high density lipoprotein; T2D, type 2 diabetes mellitus controls group; PsP+T2D, the group of type 2 diabetes mellitus with PsO.

female patients compared with male patients. ${ }^{15}$ However, these findings should be validated using a larger sample size and through other possible basic methodologies. Notably, the duration of diabetes for patients with $\mathrm{PsO}$ was significantly shorter compared with that for the T2D only group $(P=0.012<0.05$, Table 1 , Figure 2$)$. Analysis of age, BMIs, and HOMA- $\beta$ level (Figure 3) showed no significant differences between the two groups. This finding indicates that similar levels of BMIs and pancreatic islet function occur within the same age group. PsO may induce significant IR compared with patients with T2D alone. Notably, increased IR may not be solely attributed to dissimilarities in progress of T2D. In addition, the PsO group showed a slightly higher mean BMI compared with the normal BMI. Studies report that higher BMI or obesity is a critical risk factor of progression of $\mathrm{PsO}{ }^{16,17}$ These findings are consistent with findings from previous studies that patients with $\mathrm{PsO}$ are at higher risk of developing $\mathrm{T} 2 \mathrm{D},{ }^{18}$ and have a risk of developing IR. Analysis showed no significant differences in HbAlc between the groups, implying that the levels of hyperglycemia management in the two groups in the past 3 months were similar. However, the mean blood glucose level determined by HbAlc in the past 3 months was analyzed, and oral drugs like retinoic acid and methotrexate were administered to the $\mathrm{PsO}$ group during this time, which may influence regulation of blood glucose. However, a previous study reports that the effect of retinoic acid therapy is through transiently decreasing HOMA- $\beta$ and HDL level. Notably, this change was not associated with change in 
Table 2 Levels of Plasma Glucose, C-Peptide, and Insulin at 0 and 120 minutes between PsO+T2D and Control Patients

\begin{tabular}{|c|c|c|c|c|}
\hline & & PsO+T2D & Controls & $P$-value \\
\hline \multirow{2}{*}{$\begin{array}{l}\text { Plasma } \\
\text { glucose } \\
(\mathrm{mmol} / \mathrm{L})\end{array}$} & 0 min & $9.6 \pm 5.06 \mathrm{I}$ & $8.6 \pm 3.495$ & 0.481 \\
\hline & $120 \mathrm{~min}$ & $15.8 \pm 5.528$ & $14.2 \pm 5.412$ & 0.219 \\
\hline \multirow{4}{*}{$\begin{array}{l}\text { C-peptide } \\
\text { (nmol/L) }\end{array}$} & 0 min & 0.6611 & 0.3633 & $0.018^{*}$ \\
\hline & & \pm 0.440 & \pm 0.252 & \\
\hline & $120 \mathrm{~min}$ & 1.2247 & 0.8162 & $0.00 I^{* *}$ \\
\hline & & \pm 0.984 & \pm 0.613 & \\
\hline \multirow{4}{*}{$\begin{array}{l}\text { Insulin } \\
\text { (pmol/L) }\end{array}$} & 0 min & 131.726 & 129.106 & 0.998 \\
\hline & & \pm 81.041 & \pm 110.337 & \\
\hline & $120 \mathrm{~min}$ & 459.670 & 381.525 & 0.348 \\
\hline & & \pm 356.122 & \pm 270.788 & \\
\hline
\end{tabular}

Notes: $* P<0.05 ; * * P<0.01$.

Abbreviations: PsO, psoriasis; T2D, type 2 diabetes.

adipocytokines derived from adipose tissue such as adiponectin, resistin, and tumor necrosis factor alpha (TNF- $\alpha),{ }^{19}$ implying that it does not modulate systemic inflammation which is associated with IR. A previous study reports that administration of methotrexate for 6 months had no effect on HOMA-IR level despite the level of HbAlc declining significantly. This implies that these oral PsO individual drugs do not induce a significant increase in IR. In addition, analysis of different treatment paradigms of diabetes between the two groups show that the proportions of insulin replacement are relatively low in PsO group compared with the group without PsO. This can be attributed to the preserved pancreatic $\beta$-cell function, indicating that the pathogenesis of $\mathrm{PsO}$ is a systemic inflammatory process mainly affected by insulin action rather than insulin production. ${ }^{20}$

Further, analysis using HOMA-IR and HOMA- $\beta$ models showed that $\mathrm{PsO}$ may exacerbate reduction in insulin sensitivity in T2D. The findings of this study show that there is no significant difference in BMI between the two groups, which partially reflects $t$ pancreatic islet $\beta$-cell function and nutritional status of patients. ${ }^{21}$ In addition, a decrease in mean eGFR was observed in the two groups, however, no significant difference was observed. This finding implies that $\mathrm{PsO}$ may induce chronic pathological changes in the vascular systems including implications on kidney function. ${ }^{22} \mathrm{~A}$ recent study reported an increase in 24-hour microalbuminuria $(11.53 \pm 7.29$ vs $9.79 \pm 3.72$, $P<0.05)$ and 24 -hour proteinuria $(0.24 \pm 0.21$ vs 0.18 $\pm 0.09, P<0.005)$ in patients with $\mathrm{PsO}$ compared with controls without hypertension or diabetes. ${ }^{23}$ An increase in IR in PsO patients may result in progression to renal insufficiency earlier compared with diabetic patients without psoriasis. Moreover, analysis of the profile of lipids and lipoprotein in this study showed no statistical differences between the two groups. This finding implies that there is the baseline matching of the two groups, and IR resulting from systemic inflammation in the two groups were similar. However, further studies should explore lipid malfunction and adipocytokine release in T2D patients with PsO. Notably, analysis showed that HDL level was significantly lower compared with the management level required in T2D (HDL $<1 \mathrm{mmol} / \mathrm{L})$. This finding is consistent with reports from a previous study that HDL level is lower in PsO patients compared with normal controls. ${ }^{24}$ Changes in HDL-cholesterol levels have been reported in psoriasis patients. HDL and associated apolipoprotein (apo) potently suppress immune cell effector responses, and $\mathrm{PsO}$ could in turn affect HDL composition, metabolism, and function, thus promoting disease progression and increasing risk of cardiovascular diseases and infections. ${ }^{25}$ The mean level of TG in the PsO group was slightly higher compared with the recommended level, which was consistent with findings from a recent study that explored the risk biomarkers of cardiovascular diseases in $\mathrm{PsO}$ with T2D. ${ }^{26}$

Moreover, glucose metabolism related indices between two groups were explored. Analysis showed that the mean HOMA- $\beta$, glucose level, insulin concentration, and C-peptide were higher in $\mathrm{PsO}$ compared with the levels in the control group. Previous studies report that patients with $\mathrm{PsO}$ have a relatively preserved pancreatic $\beta$-cell function, but with increased IR. ${ }^{27}$ FPG levels were not significantly different between the two groups, implying that disequilibrium of blood glucose in $\mathrm{PsO}$ may not totally be attributed to impairment of pancreatic function, since FPG level is a marker for the basic function of the pancreas. ${ }^{28}$ In addition, PIN, PCP, and PPG levels were significantly higher in the $\mathrm{PsO}$ group compared with the controls, indicating the compensating state of the pancreas in PsO. Similar findings were reported by a study that used a mice model of psoriasis-like skin inflammation, which showed that a pre-diabetic phenotype displayed by compensating function of pancreas, and signs of metabolic dysfunction in subcutaneous and other multiple organs. ${ }^{29}$ This preliminary study only reports the general distributions of glucose metabolic diversity between normal T2D patients and T2D with PsO in our hospital, therefore, there 
A

HOMA- $\beta$

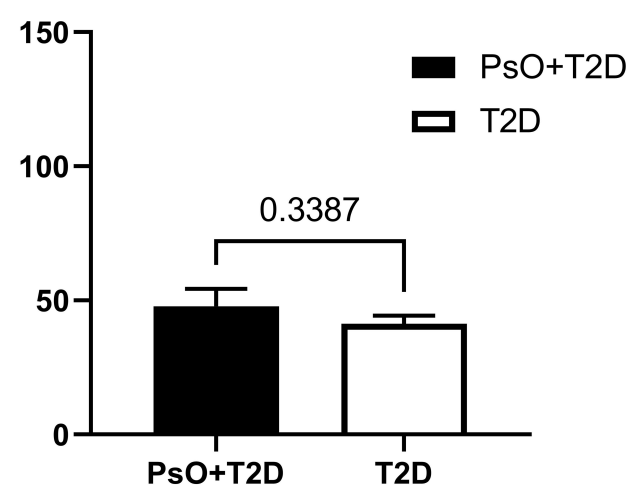

C

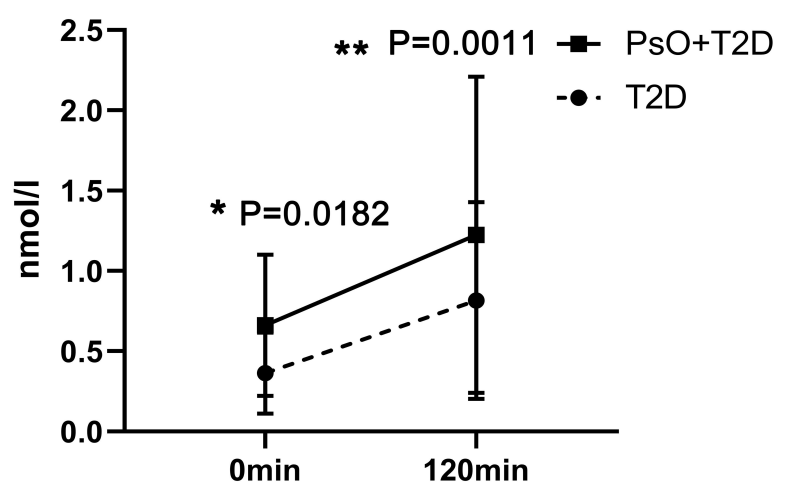

B Glucose

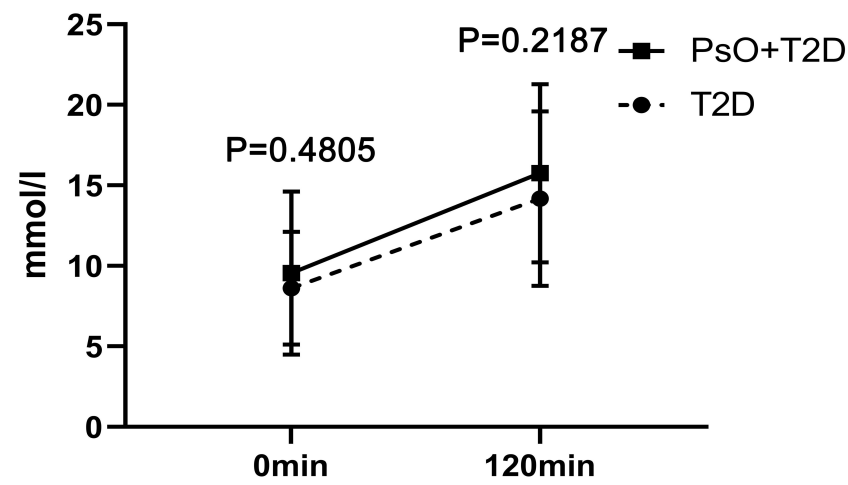

D

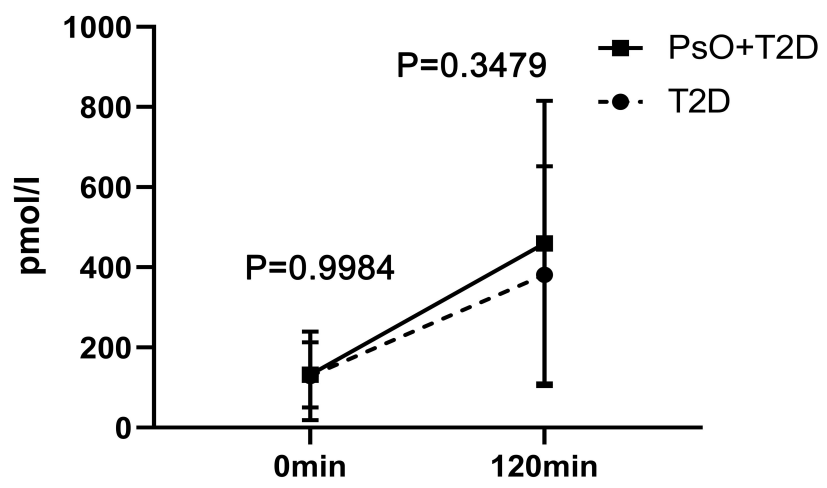

Figure 3 HOMA- $\beta$ (A), plasma glucose (B), C-peptide (C), and insulin (D) in the PsO group and control group. Notes: $* P<0.05 ; * * P<0.01$.

Abbreviations: HOMA- $\beta$, homeostasis model assessment of $\beta$-cell function state; T2D, type 2 diabetes mellitus controls group; PsP+T2D, the group of type 2 diabetes mellitus with $\mathrm{PsO}$.

is need to explore the underlying molecular and genetic mechanism of $\mathrm{PsO}$ on the pathophysiology of T2D.

$\mathrm{PsO}$ is a chronic and recurrent inflammatory skin disease caused by dysregulated interactions of innate and adaptive components of the immune system with resident cutaneous cell types. ${ }^{1}$ It is characterized by proliferation of keratinocytes in the basal layer of the epidermis and a significant increase in systemic proinflammatory factors in vivo. ${ }^{30}$ Psoriatic patients show a significantly high TNF- $\alpha$ level, and anti-TNF- $\alpha$ is an effective strategy for alleviating the pathological change of $\mathrm{PsO} .{ }^{31}$ Increase in TNF- $\alpha$ induces IR in adipocytes and peripheral tissues by impairing insulin signaling through serine phosphorylation resulting in development of T2D. Anti-TNF- $\alpha$ treatment strategies have been developed to reduce the incidence of IR and development of T2D. ${ }^{32}$ Furthermore, interleukin23/Th17 axis is critical in PsO, and several novel targeted therapies have been designed against this axis. ${ }^{33}$ Anti-IL -17 neutralizing antibodies and/or antibodies targeting Th17 cells protect high risk individuals from T2D development. ${ }^{34}$ The findings from the current study show that the skin is a vital IR target organ, and PsO may modulate glucose regulation, thus exacerbating systemic IR (Figure 4). Further molecular studies should explore the role of the skin in insulin activity and energy metabolism.

The skin is the largest organ in the human body, and is associated with insulin action and its abnormality can result in IR (IR). ${ }^{35} \mathrm{PsO}$ and T2D are chronic inflammatory diseases which are associated with metabolic syndrome. Studies report that the two diseases share common pathophysiological and genetic mechanisms. The findings of this study have potential implications in development of novel effective therapy for diabetic patients with PsO. For instance, chronic inflammation in the subcutaneous 


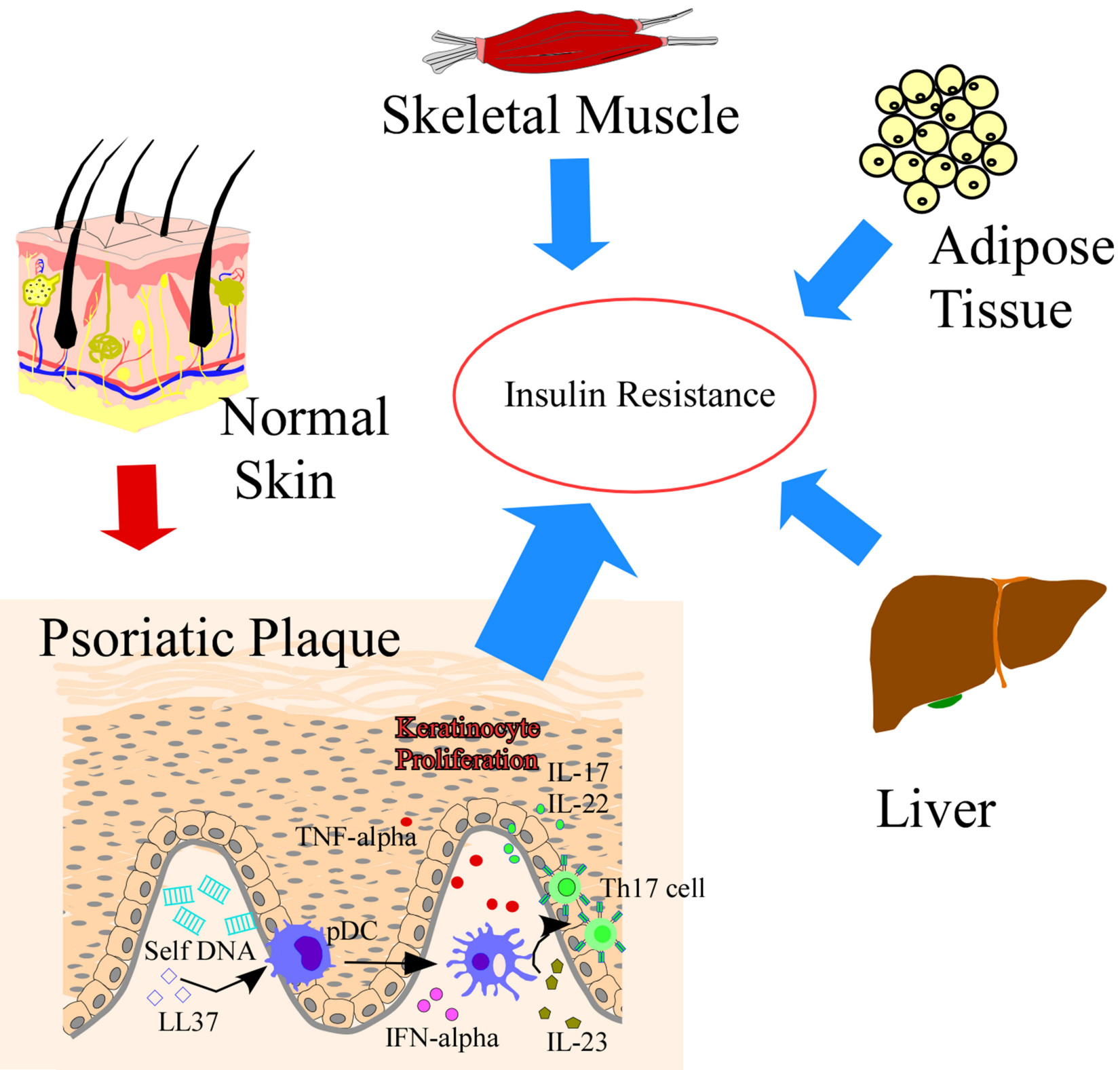

Figure 4 Possible mechanism of systemic IR and the role of skin PsO. Systemic IR can be contributed by multiple organs including skin, skeletal muscle, subcutaneous adipose tissue and liver. PsO of skin involves release of IFN- $\alpha$ by the dermal plasmacytoid dendritic cells, activation of dendritic cells which release IL-23 and TNF- $\alpha$, and activity of ThI7 cells which generate IL-I7 and IL-22. These responses eventually increase proliferation of keratinocytes and other pathological alterations, resulting in inflammation of skin and an increase in IR.

Abbreviations: LL37, cathelicidin; PDC, dermal plasmacytoid dendritic cells; IFN- $\alpha$, interferon alpha; TNF- $\alpha$, tumor necrosis factor alpha; ThI7, T helper cell I7; IL-23, interleukin 23; IL-17, interleukin I7; IL-22, interleukin 22.

adipose tissue, and pathologic change in the microvasculature may generate IR, ${ }^{36}$ which is a component of systemic IR in addition to typical insulin targets such as liver and muscle. Higher IR associated with PsO has been reported in clinical background. One case study reported that $\mathrm{PsO}$ is causally associated with type B IR, an extremely rare condition of IR caused by circulating antibodies against insulin receptor. ${ }^{37}$ Furthermore, a recent study reported that HOMA-IR is positively correlated with digital ulcer in patients with systemic sclerosis. ${ }^{38}$ Notably, low-grade inflammation in $\mathrm{PsO}$ is shown by elevated levels of high-sensitivity C-reactive protein (hCRP), ${ }^{39}$ which is consistent with our unpublished data that hCRP is significantly associated with an increased insulin dosage in T2D patients with infection. Moreover, our previous clinical findings showed that anti-hyperglycemia agents 
such as thiazolidinediones (TZD) can relieve PsO symptoms in diabetic patient and can change the chronic inflammatory status of the skin. ${ }^{40}$

This study explored the epidemiological distributions and metabolic relationships of $\mathrm{PsO}$ and T2D. The findings of this study show that PsO plays a role in IR of skin which could exacerbate glucose metabolism and affect disease progression. However, further studies should explore the specific molecular targets, or the relationship of severity of $\mathrm{PsO}$ with extent of IR. The findings of the present study show that patients with T2D with co-morbidity of PsO may present higher IR, and amelioration of inflammation in local skin may be effective in improving overall insulin sensitivity.

\section{Ethical Statement}

Ethical approval for this study including surveys, sampling and examinations was obtained from the Ethics Committee of Shanghai Pudong Hospital (2020 No.WZ-01), Fudan University (Shanghai, China). Informed written consent was obtained from patients prior to the study. Guidelines outlined and procedures used in this study were in accordance with the Declaration of Helsinki.

\section{Acknowledgment}

We should thank Dr, Xinlu Yuan, Dr. Min Gong, Dr. Congcong Wang, Dongxiang $\mathrm{Xu}$, and Dr. Chaoxun Wang who helped in patient's diagnoses, treatments, and medical records management. We should thank Dr. Jianlan Jin who was responsible for management of patients' care and assisted with project administration. The authors thank staff from Shanghai Pudong Hospital for their tremendous and numerous help during this study.

\section{Author Contributions}

All authors made a significant contribution to the work reported, whether that is in the conception, study design, execution, acquisition of data, analysis and interpretation, or in all these areas. All authors have drafted or written, or substantially revised or critically reviewed the article. All authors have agreed on the journal to which the article will be submitted. All authors reviewed and agreed on all versions of the article before submission, during revision, the final version accepted for publication, and any significant changes introduced at the proofing stage. All authors agree to take responsibility and be accountable for the contents of the article.

\section{Funding}

This work was supported by the Project of Key Medical Discipline of Pudong Hospital of Fudan University (Zdxk2020-11), the Project of Key Medical Specialty and Treatment Center of Pudong Hospital of Fudan University (Zdzk2020-24), Special Department Fund of the Pudong New Area Health Planning Commission (PWZzk2017-03), and Integrative Medicine special fund of Shanghai Municipal Health Planning Committee (ZHYYZXYJHZX-2-201712), National Natural Science Foundation of China (81370932), Outstanding Leaders Training Program of Pudong Health Bureau of Shanghai (PWR12014-06), the Outstanding Clinical Discipline Project of Shanghai Pudong (PWYgy-2018-08), the Natural Science Foundation of China (21675034) and Shanghai Natural Science Foundation (19ZR1447500).

\section{Disclosure}

The authors declare no conflicts of interest.

\section{References}

1. Boehncke W-H, Schön MP. Psoriasis. Lancet. 2015;386:983-994. doi:10.1016/s0140-6736(14)61909-7

2. Griffiths CE, Barker JN. Pathogenesis and clinical features of psoriasis. Lancet. 2007;370:263-271. doi:10.1016/s0140-6736(07) 61128-3

3. Nestle FO, Kaplan DH, Barker J. Psoriasis. $N$ Engl J Med. 2009;361:496-509. doi:10.1056/NEJMra0804595

4. American Diabetes Association. Diagnosis and classification of diabetes mellitus. Diabetes Care. 2013;36(Suppl 1):S67-S74. doi:10.2337/dc13-S067

5. Wan MT, Shin DB, Hubbard RA, et al. Psoriasis and the risk of diabetes: a prospective population-based cohort study. $J$ Am Acad Dermatol. 2018;78:315-322.e311. doi:10.1016/j.jaad.2017.10.050

6. Yeung H, Takeshita J, Mehta NN, et al. Psoriasis severity and the prevalence of major medical comorbidity: a population-based study. JAMA Dermatol. 2013;149(10):1173-1179. doi:10.1001/ jamadermatol.2013.5015

7. Quaranta M, Burden AD, Griffiths CEM, et al. Differential contribution of CDKAL1 variants to psoriasis, Crohn's disease and type II diabetes. Genes Immun. 2009;10:654-658. doi:10.1038/gene.2009.51

8. Azfar RS, Gelfand JM. Psoriasis and metabolic disease: epidemiology and pathophysiology. Curr Opin Rheumatol. 2008;20:416-422. doi:10.1097/BOR.0b013e3283031c99

9. Elder JT, Bruce AT, Gudjonsson JE, et al. Molecular dissection of psoriasis: integrating genetics and biology. $J$ Invest Dermatol. 2010;130:1213-1226. doi:10.1038/jid.2009.319

10. Kirby B, Lynch M. Adipokines and psoriasis: the obesity link. $\mathrm{Br}$ J Dermatol. 2018;179:239. doi:10.1111/bjd.16803

11. Gyldenløve M, Vilsbøll T, Zachariae C, et al. Impaired incretin effect is an early sign of glucose dysmetabolism in nondiabetic patients with psoriasis. J Intern Med. 2015;278(6):660-670. doi:10.1111/ joim. 12388

12. Al-Mutairi N, Shabaan D. Effects of tumor necrosis factor $\alpha$ inhibitors extend beyond psoriasis: insulin sensitivity in psoriasis patients with type 2 diabetes mellitus. Cutis. 2016;97:235-241. 
13. Stanley TL, Zanni MV, Johnsen S, et al. TNF- $\alpha$ antagonism with etanercept decreases glucose and increases the proportion of high molecular weight adiponectin in obese subjects with features of the metabolic syndrome. J Clin Endocrinol Metab. 2011;96:E146-E150. doi:10.1210/jc.2010-1170

14. Hägg D, Eriksson M, Sundström A, Schmitt-Egenolf M. The higher proportion of men with psoriasis treated with biologics may be explained by more severe disease in men. PLoS One. 2013;8: e63619. doi:10.1371/journal.pone.0063619

15. Maul JT, Augustin M, Sorbe C, et al. Association of gender and systemic therapy treatment outcomes in psoriasis: a two-country, multi-centre, prospective, non-interventional registry study. $\mathrm{Br}$ J Dermatol. 2021. doi:10.1111/bjd.20387

16. Kamiya K, Kishimoto M, Sugai J, Komine M, Ohtsuki M. Risk factors for the development of psoriasis. Int $J$ Mol Sci. 2019;20:4347. doi:10.3390/ijms20184347

17. Jensen P, Zachariae C, Christensen R, et al. Effect of weight loss on the cardiovascular risk profile of obese patients with psoriasis. Acta Derm Venereol. 2014;94:691-694. doi:10.2340/00015555-1824

18. Ikumi K, Odanaka M, Shime H, et al. Hyperglycemia is associated with psoriatic inflammation in both humans and mice. $J$ Invest Dermatol. 2019;139:1329-1338.e1327. doi:10.1016/j.jid.2019.01.029

19. Corbetta S, Angioni R, Cattaneo A, Beck-Peccoz P, Spada A. Effects of retinoid therapy on insulin sensitivity, lipid profile and circulating adipocytokines. Eur J Endocrinol. 2006;154:83-86. doi:10.1530/ eje. 1.02057

20. Gyldenlove M, Storgaard H, Holst JJ, et al. Patients with psoriasis are insulin resistant. $J$ Am Acad Dermatol. 2015;72:599-605. doi:10.1016/j.jaad.2015.01.004

21. Ko SH, Chi CC, Yeh ML, et al. Lifestyle changes for treating psoriasis. Cochrane Database Syst Rev. 2019;7:Cd011972. doi:10.1002/14651858.CD011972.pub2

22. Visconti L, Leonardi G, Buemi M, et al. Kidney disease and psoriasis: novel evidences beyond old concepts. Clin Rheumatol. 2016;35:297-302. doi:10.1007/s10067-015-3126-4

23. Ren F, Zhang M, Hao L, Sang H. Kidney involvement in psoriasis: a case-control study from China. Int Urol Nephrol. 2017;49:1999-2003. doi:10.1007/s11255-017-1692-x

24. Reynoso-von Drateln C, Martinez-Abundis E, Balcazar-Munoz BR, Bustos-Saldana R, Gonzalez-Ortiz M. Lipid profile, insulin secretion, and insulin sensitivity in psoriasis. $J$ Am Acad Dermatol. 2003;48:882-885. doi:10.1067/mjd.2003.446

25. Trakaki A, Marsche G. High-density lipoprotein (HDL) in allergy and skin diseases: focus on immunomodulating functions. Biomedicines. 2020;8(12):558. doi:10.3390/biomedicines8120558

26. Brazzelli V, Maffioli P, Bolcato V, et al. Psoriasis and diabetes, a dangerous association: evaluation of insulin resistance, lipid abnormalities, and cardiovascular risk biomarkers. Front Med. 2021;8:605691. doi:10.3389/fmed.2021.605691
27. Ucak S, Ekmekci TR, Basat O, Koslu A, Altuntas Y. Comparison of various insulin sensitivity indices in psoriatic patients and their relationship with type of psoriasis. J Eur Acad Dermatol Venereol. 2006;20:517-522. doi:10.1111/j.1468-3083.2006.01499.x

28. Zhang Q, Zhao G, Yang N, Zhang L. Fasting blood glucose levels in patients with different types of diseases. Prog Mol Biol Transl Sci. 2019;162:277-292. doi:10.1016/bs.pmbts.2019.01.004

29. Evans EA, Sayers SR, Kodji X, et al. Psoriatic skin inflammation induces a pre-diabetic phenotype via the endocrine actions of skin secretome. Mol Metab. 2020;41:101047. doi:10.1016/j. molmet.2020.101047

30. Rendon A, Schäkel K. Psoriasis pathogenesis and treatment. Int J Mol Sci. 2019;20(6):1475. doi:10.3390/ijms20061475

31. Campa M, Ryan C, Menter A. An overview of developing TNF- $\alpha$ targeted therapy for the treatment of psoriasis. Expert Opin Investig Drugs. 2015;24:1343-1354. doi:10.1517/13543784.2015.1076793

32. Akash MSH, Rehman K, Liaqat A. Tumor necrosis factor-alpha: role in development of insulin resistance and pathogenesis of type 2 diabetes mellitus. J Cell Biochem. 2018;119:105-110. doi:10.1002/ jcb.26174

33. Di Cesare A, Di Meglio P, Nestle FO. The IL-23/Th17 axis in the immunopathogenesis of psoriasis. $J$ Invest Dermatol. 2009;129:1339-1350. doi:10.1038/jid.2009.59

34. Abdel-Moneim A, Bakery HH, Allam G. The potential pathogenic role of IL-17/Th17 cells in both type 1 and type 2 diabetes mellitus. Biomed Pharmacother. 2018;101:287-292. doi:10.1016/j. biopha.2018.02.103

35. Hu Y, Zhu Y, Lian N, et al. Metabolic syndrome and skin diseases. Front Endocrinol. 2019;10:788. doi:10.3389/fendo.2019.00788

36. Gong M, Wen S, Nguyen T, et al. Converging relationships of obesity and hyperuricemia with special reference to metabolic disorders and plausible therapeutic implications. Diabetes Metab Syndr Obes. 2020;13:943-962. doi:10.2147/dmso.s232377

37. Łebkowska A, Krentowska A, Adamska A, et al. Type B insulin resistance syndrome associated with connective tissue disease and psoriasis. Endocrinol Diabetes Metab Case Rep. 2020;2020. doi:10.1530/edm-20-0027

38. Park EK, Lee SG, Kim BH, et al. Insulin resistance is associated with digital ulcer in patients with systemic sclerosis. Clin Exp Rheumatol. 2016;34(Suppl 100):85-91.

39. Wang X, Bao W, Liu J, et al. Inflammatory markers and risk of type 2 diabetes: a systematic review and meta-analysis. Diabetes Care. 2013;36:166-175. doi:10.2337/dc12-0702

40. Zhu S. Effect of thiazolidinedione on a Patient of psoriasis combined with type 2 diabetes mellitus: acase report. Chin J Endocrinol Metab. $2018 ; 34$.

Diabetes, Metabolic Syndrome and Obesity: Targets and Therapy

Dovepress

\section{Publish your work in this journal}

Diabetes, Metabolic Syndrome and Obesity: Targets and Therapy is an international, peer-reviewed open-access journal committed to the rapid publication of the latest laboratory and clinical findings in the fields of diabetes, metabolic syndrome and obesity research. Original research, review, case reports, hypothesis formation, expert opinion and commentaries are all considered for publication. The manuscript management system is completely online and includes a very quick and fair peer-review system, which is all easy to use. Visit http://www.dovepress.com/testimonials.php to read real quotes from published authors. 